\title{
A CLASSIC MORITA EQUIVALENCE RESULT FOR FELL BUNDLE $C^{*}$-ALGEBRAS
}

\author{
MARIUS IONESCU and DANA P. WILLIAMS*
}

\begin{abstract}
We show how to extend a classic Morita Equivalence Result of Green's to the $C^{*}$-algebras of Fell bundles over transitive groupoids. Specifically, we show that if $p: \mathscr{B} \rightarrow G$ is a saturated Fell bundle over a transitive groupoid $G$ with stability group $H=G(u)$ at $u \in G^{(0)}$, then $C^{*}(G, \mathscr{B})$ is Morita equivalent to $C^{*}(H, \mathscr{C})$, where $\mathscr{C}=\left.\mathscr{B}\right|_{H}$. As an application, we show that if $p: \mathscr{B} \rightarrow G$ is a Fell bundle over a group $G$ and if there is a continuous $G$-equivariant map $\sigma$ : Prim $A \rightarrow G / H$, where $A=B(e)$ is the $C^{*}$-algebra of $\mathscr{B}$ and $H$ is a closed subgroup, then $C^{*}(G, \mathscr{B})$ is Morita equivalent to $C^{*}\left(H, \mathscr{C}^{I}\right)$ where $\mathscr{C}^{I}$ is a Fell bundle over $H$ whose fibres are $A / I-A / I$-imprimitivity bimodules and $I=\bigcap\{P: \sigma(P)=e H\}$. Green's result is a special case of our application to bundles over groups.
\end{abstract}

\section{Introduction}

One of the many fundamental results in Green's seminal work [3] on $C^{*}$ dynamical systems is his theorem ([3, Theorem 17]) which says that if $(A, G, \alpha)$ is a dynamical system and if $\sigma: \operatorname{Prim} A \rightarrow G / H$ is a continuous $G$ equivariant map, then $A \rtimes_{\alpha} G$ is Morita equivalent to $A / I \rtimes_{\alpha^{I}} H$, where $I=\bigcap\{P \in \operatorname{Prim} A: \sigma(P)=e H\}$. This result is of particular importance in studying the Mackey machine for regular or smooth crossed products - see [10, Proposition 8.7 and Theorem 8.16] - and consequently is a basic component of the Mackey machine in general. Our first goal in this note is to show how Green's result can be formulated for Fell bundles where it is a straightforward application of the Equivalence Theorem [7, Theorem 6.4]. However, recovering Green's dynamical system version from the Fell bundle version is nontrivial. Fortuitously, doing so leads to an interesting application to Fell bundles over groups which is our second main result.

This note is a continuation of [4]. In particular, we will refer to the first section of that paper for basic notation, conventions and some fundamental facts about Fell bundles. In particular, in order that we can apply the Equivalence Theorem from [7], we are going to want to assume that our Fell bundles are

\footnotetext{
* This research was supported by the Edward Shapiro fund at Dartmouth College.

Received 12 February 2010.
} 
saturated and separable in the sense that the underlying groupoids are second countable and the underlying Banach bundles are separable.

\section{The Main Results}

The first order of business is to formulate Green's theorem for Fell bundles. Then, as mentioned above, we have some work to do to extract the "group version".

\subsection{The Fell Bundle Version}

THEOREM 1. Let $p: \mathscr{B} \rightarrow G$ be a separable saturated Fell bundle over a transitive locally compact groupoid $G$. If $u \in G^{(0)}$ and if $H:=G(u)=\{x \in$ $G: s(x)=u=r(x)\}$ is the stability group at $u$, then $\mathscr{C}=p^{-1}(H)$ is a Fell bundle over $H$, and $C^{*}(G, \mathscr{B})$ is Morita equivalent to $C^{*}(H, \mathscr{C})$.

Remark 2. Since we have assumed that $p: \mathscr{B} \rightarrow G$ is separable, this implies that $G$ is second countable. Therefore $G_{u}=s^{-1}(u)$ is a $(G, H)$ equivalence under the hypotheses of Theorem 1 (see [6, Theorem 2.2B]). ${ }^{1}$ In particular, $C^{*}(G)$ and $C^{*}(H)$ are Morita equivalent by the Equivalence Theorem for groupoids ([6, Theorem 2.8]).

Proof. Let $\mathscr{C}=p^{-1}(H)$ and $p_{H}=\left.p\right|_{\mathscr{C}}$. Then it is straightforward to see that $p_{H}: \mathscr{C} \rightarrow H$ is a Fell bundle over $H$. Let $\mathscr{E}=p^{-1}\left(G_{u}\right)$ and $q=\left.p\right|_{\mathscr{E}}$. We will show that $q: \mathscr{E} \rightarrow G_{u}$ is a $(\mathscr{B}, \mathscr{C})$-equivalence as in [7, Definition 6.1]. Then Theorem 1 will follow from the Equivalence Theorem [7, Theorem 6.4].

Since $G_{u}$ is a $(G, H)$-equivalence (Remark 2$)$ and $q: \mathscr{E} \rightarrow G_{u}$ is clearly an upper semicontinuous-Banach bundle, we just need to verify axioms (a), (b) and (c) of [7, Definition 6.1]. To do this, first observe that $\mathscr{B}$ and $\mathscr{C}$ act on the left and right, respectively, on $\mathscr{E}$ via restriction of the multiplication in $\mathscr{B}^{(2)}$; then the axioms (a), (b) and (c) for an action given in the second paragraph of [7, §6] are clearly satisfied. ${ }^{2}$ Then axiom (a) of [7, Definition 6.1] follows from the associativity of multiplication in $\mathscr{B}^{(2)}$.

For axiom (b) of [7, Definition 6.1], we define $\mathscr{B}_{\mathscr{B}}\langle\cdot, \cdot\rangle: \mathscr{E} *_{s} \mathscr{E} \rightarrow \mathscr{B}$ by

$$
{ }_{\mathscr{B}}\langle b, c\rangle=b c^{*},
$$

\footnotetext{
${ }^{1}$ The only issue in showing that $G_{u}$ is an equivalence is to prove that $\left.r_{G}\right|_{G_{u}}$ is an open map. (Note that this can fail if $G$ is not second countable: consider R with the discrete topology acting on $\mathrm{R}$ with the usual topology by translation.) The point of [6, Theorem 2.2B] is to see that this map is always open if $G$ is second countable. Nowadays, a better reference for this is Ramsay's [9, Theorem 2.1].

${ }^{2}$ Notice that there is a typo in axiom (c): it should read $\|b \cdot e\| \leq\|b\|\|e\|$. Of course, this follows from [4, Lemma 1] in our case.
} 
and $\langle b, c\rangle_{\mathscr{E}}: \mathscr{E} *_{r} \mathscr{E} \rightarrow \mathscr{C}$ by

$$
\langle b, c\rangle_{\mathscr{C}}=b^{*} c .
$$

Then it is not hard to check that properties (i)-(iv) hold. For example, if $(b, c) \in$ $\mathscr{E} *_{s} \mathscr{E}$, then $[q(b), q(c)]=q(b) q(c)^{-1}=p\left(b c^{*}\right)$.

As for axiom (c), let $A=\Gamma_{0}\left(G^{(0)} ; \mathscr{B}\right)$ the $C^{*}$-algebra of $\mathscr{B}$ over $G^{(0)}$. We are given that $E(x)=B(x)$ is a $A(r(x))-A(u)$-imprimitivity bimodule. But $A(u)$ is the $C^{*}$-algebra of $\mathscr{C}$ over $H^{(0)}=u$. Thus, (c) holds and $q: \mathscr{E} \rightarrow G_{u}$ is an equivalence. ${ }^{3}$ This completes the proof.

\subsection{The Group Version}

It is hardly obvious that Green's Theorem for $C^{*}$-dynamical systems is a consequence of Theorem 1. In fact, showing this requires a fair bit of gymnastics. We will obtain Green's result as a special case of a result for Fell bundles over groups (Theorem 3 ) that is of considerable interest in its own right.

Let $p: \mathscr{B} \rightarrow G$ be a Fell bundle over a locally compact group. (Note that this implies that the underlying Banach bundle is continuous rather than merely upper semicontinuous - see [4, Remark 3].) Suppose that $\sigma: \operatorname{Prim} A \rightarrow G / H$ is an equivariant map, where $A$ is the $C^{*}$-algebra $A(e)$ of $\mathscr{B}$ over $G^{(0)}=\{e\}$, and $H$ is a closed subgroup of $G$. We let

$$
I:=\bigcap\{P \in \operatorname{Prim} A: \sigma(P)=e H\} .
$$

As in Theorem 1, we let $\mathscr{C}=p^{-1}(H)$. Then $I$ is a $H$-invariant ideal in the $C^{*}$ algebra $A=A(e)$ of $\mathscr{C}$. We adopt the notations and constructions of $[4, \S 3.1]$ applied to the Fell bundle $\left.p\right|_{\mathscr{C}}: \mathscr{C} \rightarrow G$ and the invariant ideal $I$. In particular, for each $h \in H, C(h)$ is an $A-A$-imprimitivity bimodule, and $C_{I}(h):=C(h) \cdot I$ is an $I-I$-imprimitivity bimodule by [4, Lemma 10 and Proposition 14]. Thus by [8, Proposition 3.25], the quotient $C^{I}(h):=C(h) / C_{I}(h)$ is an $A / I-A / I-$ imprimitivity bimodule, and by [4, Proposition 15], $\mathscr{C}^{I}:=\bigsqcup_{h \in H} C^{I}(h)$ has a natural topology making it into a Fell bundle over $H$ with the operations induced from $\mathscr{C}$.

THEOREM 3. Let $p: \mathscr{B} \rightarrow G$ be a separable saturated Fell bundle over a locally compact group $G$ such that there is a continuous $G$-equivariant map $\sigma: \operatorname{Prim} A \rightarrow G / H$, where $A$ is the $C^{*}$-algebra of $\mathscr{B}$ and $H$ is a closed subgroup of $G$. If I is the ideal of A given in (1), and if $\mathscr{C}$ is the Fell bundle $p^{-1}(H)$ as above, then $C^{*}(G, \mathscr{B})$ is Morita equivalent to $C^{*}\left(H, \mathscr{C}^{I}\right)$

\footnotetext{
${ }^{3}$ There is, sadly, also a misprint in part (c) of [7, Definition 6.1]: it should read that "each $E(t)$ is a $B(r(t))-C(s(t))$-imprimitivity bimodule."
} 
Remark 4. Notice that if $\mathscr{B}=A \times G$ is the Fell bundle associated to the dynamical system $(A, G, \alpha)$, then $\mathscr{C}^{I}$ is the Fell bundle $A / I \times H$ associated to $\left(A / I, H, \alpha^{I}\right)$. Therefore Green's Theorem is a special case of Theorem 3 .

To prove Theorem 3 we want to appeal to Theorem 1 . We do this by first building a Fell bundle $\mathscr{Q}$ over the transitive transformation groupoid $G \times G / H$ such that if $u=(e, e H) \in(G \times G / H)^{(0)}$ and if $\underline{H}=\{(h, e H): h \in$ $H\}$ is the stability group at $u$, then $\left.\mathscr{2}\right|_{\underline{H}}$, which we view as a Fell bundle over $H$, is naturally identified with $\mathscr{C}^{I}$ (see Proposition 13). Since Theorem 1 implies $C^{*}(G \times G / H, \mathscr{Q})$ is Morita equivalent to $\mathscr{C}^{I}$, we complete the proof of Theorem 3 by showing that $C^{*}(G \times G / H, \mathscr{Q})$ is isomorphic to $C^{*}(G, \mathscr{B})$. We do this in the next section as Proposition 15.

\section{The isomorphism}

Suppose that we are given a Fell bundle $p: \mathscr{B} \rightarrow G$ over a locally compact group $G$. Let $A=B(e)$ be the $C^{*}$-algebra over $e$. In [4, Proposition 9], we showed that Prim $A$ is a $G$-space. Suppose that there is a $G$-equivariant map $\sigma: \operatorname{Prim} A \rightarrow G / H$ for a closed subgroup $H$ of $G$. In this section, we want to show that there is a naturally associated Fell bundle $\bar{p}: \mathscr{Q} \rightarrow G \times G / H$ over the transformation groupoid $G \times G / H$ such that $C^{*}(G, \mathscr{B})$ and $C^{*}(G \times G / H, \mathscr{Q})$ are isomorphic (Proposition 15).

Lemma 5. Let $\sigma: \operatorname{Prim} A \rightarrow G / H$ be a continuous $G$-equivariant map as above. Then $A$ is a $C_{0}(G / H)$-algebra (as in [10, Proposition C.5]) and $A(x H)=A / I(x H)$, where

$$
I(x H):=\bigcap\{P \in \operatorname{Prim} A: \sigma(P)=x H\} .
$$

Proof. By [10, Proposition C.5] and preceding discussion, $A$ is a $C_{0}(G / H)$ algebra with fibres $A(x H)=A / J_{x H}$, where

$$
J_{x H}=\overline{\operatorname{span}}\left\{\varphi \cdot a: a \in A, \varphi \in C_{0}(G / H) \text { and } \varphi(x H)=0\right\} .
$$

Thus, we just need to confirm that $J_{x H}=I(x H)$. However, if $a(P)$ denotes the image of $a \in A$ in the quotient $A / P$, then for all $P \in \operatorname{Prim} A$,

$$
(\varphi \cdot a)(P)=\varphi(\sigma(P)) a(P)
$$

(see the discussion preceding [10, Proposition C.5]). If $P \supset J_{x H}$, then since the left-hand side of (2) vanishes for all $a \in A$ and $\varphi \in C_{0}(G / H)$ with $\varphi(x H)=0$, we see that $\sigma(P)=x H$. On the other hand, if $\sigma(P)=x H$, then using (2), we see that $J_{x H} \subset P$. Therefore $J_{x H}=I(x H)$ as required. 
Corollary 6. For each $x \in G$, let $h_{x}: \mathscr{I}(A) \rightarrow \mathscr{I}(A)$ be the Rieffel homeomorphism $B(x)$-Ind induced by the imprimitivity bimodule $B(x)$ (see [8, Proposition 3.24]). Then $h_{x}(I(y H))=I(x y H)$.

Proof. Since $\sigma$ is continuous, $\sigma^{-1}(y H)$ is closed in Prim $A$. In particular, $P \supset I(y H)$ if and only if $\sigma(P)=y H$. Since $h_{x}$ is containment preserving and has inverse $h_{x^{-1}}$ by [8, Theorem 3.29], $P \supset I(y H)$ if and only if $h_{x}(P) \supset$ $h_{x}(I(y H))$. But equivariance means that $\sigma\left(h_{x}(P)\right)=x \cdot \sigma(P)$. Thus

$$
\begin{aligned}
h_{x}(I(y H)) & =\bigcap\left\{h_{x}(P): \sigma(P)=y H\right\} \\
& =\bigcap\{P: \sigma(P)=x y H\} \\
& =I(x y H) .
\end{aligned}
$$

Remark 7. As noted in [4, Remark 5], if $X$ is a $A-B$-imprimitivity bimodule and $J$ is an ideal in $A$, then the Cohen Factorization Theorem implies that

$$
\overline{\operatorname{span}}\{a \cdot x: a \in J \text { and } x \in X\}=\{a \cdot x: a \in J \text { and } x \in X\} .
$$

Consequently, we write simply $J \cdot X$ for the above $A-B$-submodule. Similarly, we'll write $X \cdot I$ for the corresponding $A-B$-submodule when $I$ is an ideal in $B$.

For each $x \in G, B(x)$ is an $A$-A-imprimitivity bimodule. Since $I(y H)$ and $I(x y H)$ are matched up by the Rieffel correspondence, the following is a consequence of basic Morita theory (see [8, Propositions 3.24 and 3.25]).

Corollary 8. Let $x, y \in G$. Then

(a) $B(x) \cdot I(y H)=I(x y H) \cdot B(x)$,

(b) $B(x) \cdot I(y H)$ is a $I(x y H)-I(y H)$-imprimitivity bimodule, and

(c) $B(x) / B(x) \cdot I(y H)$ is $A(x y H)-A(y H)$-imprimitivity bimodule.

It follows immediately from Corollary 8 that the Banach space

$$
Q(x, y H):=B(x) / B(x) \cdot I\left(x^{-1} y H\right)=B(x) / I(y H) \cdot B(x)
$$

is an $A(y H)-A\left(x^{-1} y H\right)$-imprimitivity bimodule. Note that the $A(y H)$-valued inner product on $Q(x, y H)$ is given by taking the appropriate quotient of the left $A$-valued inner product on $B(x)$ which is given by the Fell bundle multiplication. Thus

$$
{ }_{A(y H)}\langle[b],[c]\rangle=b c^{*}(y H),
$$


where [b] denotes the image of $b \in B(x)$ in $Q(x, y H)$, and $b c^{*}(y H)$ is the image of $b c^{*}$ in $A(y H)$.

Let

$$
\mathscr{Q}:=\coprod_{(x, y H) \in G \times G / H} Q(x, y H)
$$

and $\bar{p}: \mathscr{Q} \rightarrow G \times G / H$ be the associated bundle. Naturally, we want to equip 2 with a topology and operations making it into a Fell bundle over the transformation groupoid $G \times G / H$. This will take a bit of work and will be accomplished in Proposition 13.

If $f \in \Gamma_{c}(G ; \mathscr{B})$, then let $\Phi(f)(x, y H)$ denote the image of $f(x)$ in $Q(x, y H)$.

Lemma 9. If $f \in \Gamma_{c}(G ; \mathscr{B})$, then $(x, y H) \mapsto\|\Phi(f)(x, y H)\|$ is upper semicontinuous and vanishes at infinity on $G \times G / H$.

Proof. Since $Q(x, y H)$ is an imprimitivity bimodule, and in view of (3), we have

$$
\|\Phi(f)(x, y H)\|^{2}=\left\|f(x) f(x)^{*}(y H)\right\| .
$$

Therefore it suffices to show that $(x, y H) \mapsto\|a(x)(y H)\|$ is upper semicontinuous and vanishes at infinity for $a \in C_{c}(G, A)$.

To show upper semicontinuity, it suffices to show that if $\left(x_{i}, y_{i} H\right) \rightarrow$ $(x, y H)$ and $\left\|a\left(x_{i}\right)\left(y_{i} H\right)\right\| \geq \epsilon>0$ for all $i$, then we also have $\|a(x)(y H)\| \geq$ $\epsilon$. If $\|a(x)(y H)\|<\epsilon$, then we can find $\varphi \in C_{c}(G / H)$ such that $\varphi$ is identically one in a neighborhood of $y H$ and such that $\|\varphi \cdot a(x)\|<\epsilon$. Since we can assume that $\varphi\left(y_{i} H\right)=1$ for all $i$, we certainly have $\left\|a\left(x_{i}\right)\right\| \geq \epsilon$. But this contradicts the fact that $x \mapsto\|a(x)\|$ is continuous.

To see that $(x, y H) \mapsto\|a(x)(y H)\|$ vanishes at infinity, suppose that $\left\|a\left(x_{i}\right)\left(y_{i} H\right)\right\| \geq \epsilon>0$ for all $i$. It will suffice to see that $\left\{\left(x_{i}, y_{i} H\right)\right\}$ has a convergent subsequence. Since $a$ has compact support, we can pass to a subsequence, relabel, and assume that $x_{i} \rightarrow x$. Then by continuity, we can assume that $\left\|a(x)\left(y_{i} H\right)\right\| \geq \epsilon / 2$ for large $i$. Since $A$ is a $C_{0}(G / H)$-algebra, $y H \mapsto\|a(x)(y H)\|$ must vanish at infinity [10, Proposition C.10(a)]. Therefore $\left\{y_{i} H\right\}$ must have a convergent subsequence. This completes the proof.

As in [5, Lemma 1.2] and [4, §3.2], there is a nondegenerate homomorphism $\iota: A \rightarrow M\left(C^{*}(G, \mathscr{B})\right)$ such that $\iota(a) f(x)=a f(x)$. Then

$$
\Phi(\iota(a) f)(x, y H)=a(y H) \cdot \Phi(f)(x, y H) .
$$

Since $\iota$ is nondegenerate, it extends to $M(A)$, and by composition with the $C_{0}(G / H)$-structure map of $C_{0}(G / H)$ into the center of $M(A)$, we get a map 
$\hat{\imath}: C_{0}(G / H) \rightarrow M\left(C^{*}(G, \mathscr{B})\right)$. We'll write $\psi \cdot f$ in place of $\hat{\imath}(\psi) f$. Note that

$$
\Phi(\psi \cdot f)(x, y H)=\psi(y H) \Phi(f)(x, y H) .
$$

REMARK 10. If $p: \mathscr{B} \rightarrow X$ is an upper semicontinuous-Banach bundle and $f$ is a not necessarily continuous section such that there are $f_{i} \in \Gamma_{0}(X ; \mathscr{B})$ converging uniformly to $f$, then $f \in \Gamma_{0}(X ; \mathscr{B})$.

Lemma 11. If $f \in \Gamma_{c}(G ; \mathscr{B})$ and $\theta \in C_{0}(G \times G / H)$, then there is a section $\theta \cdot f \in \Gamma_{c}(G ; \mathscr{B})$ such that

$$
\Phi(\theta \cdot f)(x, y H)=\theta(x, y H) \Phi(f)(x, y H) .
$$

Proof. Notice that if $b \in B(x)$, then

$$
\|b\|^{2}=\left\|b b^{*}\right\|=\sup _{y H \in G / H}\left\|b b^{*}(y H)\right\|=\sup _{y H \in G / H}\|b(y H)\|^{2},
$$

where $b(y H)$ denotes the image of $b$ in the quotient $Q(x, y H)$. (Note that with this notation, $\Phi(f)(x, y H)$ can also be written as $f(x)(y H)$.) Also, if $\theta(x, y H)=(\omega \otimes \varphi)(x, y H):=\omega(x) \varphi(y H)$, for $\omega \in C_{0}(G)$ and $\varphi \in$ $C_{0}(G / H)$, then we can define a continuous section $\theta \cdot f$ by $\theta \cdot f(x):=$ $\omega(x)(\varphi \cdot f)(x)$ (because scalar multiplication is continuous from $\mathrm{C} \times \mathscr{B} \rightarrow \mathscr{B}$ ). Now suppose that we have a finite sum $\sum_{i} \omega_{i} \otimes \varphi_{i}$ of such functions. Then

$$
\begin{aligned}
\left\|\sum_{i}\left(\omega_{i} \otimes \varphi_{i}\right) \cdot f\right\| & =\sup _{x \in G}\left\|\sum_{i} \omega_{i}(x) \varphi_{i} \cdot f(x)\right\| \\
& =\sup _{x \in G} \sup _{y H \in G / H}\left\|\sum_{i} \omega_{i}(x) \varphi_{i}(y H) f(x)(y H)\right\| \\
& \leq\left\|\sum_{i} \omega_{i} \otimes \varphi_{i}\right\|_{\infty} \sup _{x \in G} \sup _{y H \in G / H}\|f(x)(y H)\| \\
& =\left\|\sum_{i} \omega_{i} \otimes \varphi_{i}\right\|_{\infty}\|f\| .
\end{aligned}
$$

This shows that $\theta \cdot f$ is a well defined element of $\Gamma_{c}(G ; \mathscr{B})$ provided $\theta \in$ $C_{0}(G \times G / H)$ is a finite sum as above.

If we let $\mathscr{Q}(x):=\bigsqcup_{y H \in G / H} Q(x, y H)$, then using [4, Theorem 2] and (4), we get an upper semicontinuous-Banach bundle $\bar{p}_{x}: \mathscr{2}(x) \rightarrow G / H$ such that $\Gamma:=\{y H \mapsto b(y H): b \in B(x)\}$ are continuous sections in $\Gamma_{0}(G / H ; \mathscr{2}(x))$. Since $f \in \Gamma$ implies $\varphi \cdot f \in \Gamma$ for any $\varphi \in C_{0}(G / H)$, it follows that $\Gamma$ is uniformly dense in $\Gamma_{0}(G / H ; 2(x))$ (see [7, Lemma A.4] for example). Therefore $\Phi_{x}: B(x) \rightarrow \Gamma_{0}(G / H ; \mathscr{Q}(x))$, defined by $\Phi_{x}(b)(y H)=$ 
$b(y H)$, is an isometric isomorphism. In particular, if $b \in B(x)$ and $\varphi \in$ $C_{0}(G / H)$ then there is a $\varphi \cdot b \in B(x)$ such that $(\varphi \cdot b)(y H)=\varphi(y H) b(y H)$.

Now suppose that $\theta$ is an arbitrary element of $C_{0}(G \times G / H)$ and $f \in$ $\Gamma_{c}(G ; \mathscr{B})$. In view of the above, for each $x \in G$, there is a $b(x) \in B(x)$ such that

$$
b(x)(y H)=\theta(x, y H) f(x)(y H) \quad \text { for all } \quad y H \in G / H .
$$

So it only remains to see that $x \mapsto b(x)$ is continuous.

Let $\left\{\theta_{i}\right\}$ be a sequence of functions, which are elementary sums as in (5), such that $\theta_{i} \rightarrow \theta$ uniformly. Note that there are $g_{i} \in \Gamma_{c}(G ; \mathscr{B})$ such that

$$
g_{i}(x)(y H)=\theta_{i}(x, y H) f(x)(y H) \quad \text { for all } \quad x \in G \text { and } y H \in G / H .
$$

Computing just as in (5), we have

$$
\left\|g_{i}(x)-b(x)\right\| \leq\left\|\theta_{i}-\theta\right\|_{\infty}\|f(x)\| .
$$

Thus, by Remark 10, $x \mapsto b(x)$ defines an element of $\Gamma_{c}(G ; \mathscr{B})$ as required, and the lemma is proved.

Now let

$$
\begin{aligned}
\Gamma_{c c}(G ; \mathscr{B}):=\left\{f \in \Gamma_{c}(G ; \mathscr{B}): \Phi(f)\right. \text { vanishes } \\
\quad \text { off a compact set in } G \times G / H\} .
\end{aligned}
$$

It is a consequence of Lemmas 9 and 11 that $\Gamma:=\left\{\Phi(f): f \in \Gamma_{c c}(G ; \mathscr{B})\right\}$ satisfies the requirements of [4, Theorem 2]. Thus we can equip $\bar{p}: \mathscr{Q} \rightarrow$ $G \times G / H$ with the structure of an upper semicontinuous-Banach bundle over $G \times G / H$ such that $\Gamma \subset \Gamma_{c}(G \times G / H ; 2)$.

Remark 12 (Comments on Definitions). We equip $G \times G / H$ with the usual Haar system where we identify $(G \times G / H)^{(0)}$ with $G / H$ and define $\left\{\lambda^{y H}\right\}_{y H \in G / H}$ by

$$
\lambda^{y H}(g)=\int_{G} g(x, y H) d x .
$$

There is however a subtlety in defining $C^{*}(G ; \mathscr{B})$. Here, to make the proof of Proposition 13 more elegant, we are going to treat $G$ as a groupoid. The point is that then the involution on $\Gamma_{c}(G ; \mathscr{B})$ is given by $f^{*}(x)=f\left(x^{-1}\right)^{*}$. It is often more natural when working with a Fell bundle over a group to use the involution used by Fell \& Doran in [1], [2] where $f^{*}(x)=\Delta\left(x^{-1}\right) f\left(x^{-1}\right)^{*}$ and $\Delta$ is the modular function on the group $G$. For example, when using the second formulation, it is much easier to see that one recovers the usual group 
$C^{*}$-algebra and crossed product constructions as special cases. Fortunately, the isomorphism class of $C^{*}(G, \mathscr{B})$ is unaffected by our choice - see [5, Remark 1.5].

Proposition 13. As above, if $b \in \mathscr{B}$, let $b(y H)$ be the image of $b$ in $Q(p(b), y H)$. Then if $(m, n) \in \mathscr{Q}^{(2)}=\{(m, n) \in \mathscr{Q} \times \mathscr{Q}: \bar{p}(m)=\bar{p}(n)\}$, it follows that $(m, n)$ is of the form $\left(b(y H), b^{\prime}\left(p(b)^{-1} y H\right)\right)$ for $b, b^{\prime} \in \mathscr{B}$. Then we get a well-defined map from $\mathscr{2}^{(2)}$ to $\mathscr{Q}$ by $b(y H) b^{\prime}\left(p(b)^{-1} y H\right):=$ $b b^{\prime}(y H)$. We can also get a well defined involution from 2 to 2 via $b(y H)^{*}=$ $b^{*}\left(p(b)^{-1} y H\right)$. Then, with respect to these operations, $\bar{p}: \mathscr{Q} \rightarrow G \times G / H$ is a Fell bundle. Furthermore, $\left.2\right|_{\underline{H}}$ and $\mathscr{C}^{I}$ are isomorphic as Fell bundles over $H$.

Proof. If $(m, n) \in \mathscr{Q}^{(2)}$, then $(\bar{p}(m), \bar{p}(n)) \in(G \times G / H)^{(2)}$. Thus for appropriate $x, y, z \in G$, we must have $\bar{p}(m)=(x, y H)$ and $\bar{p}(n)=\left(z, x^{-1} y H\right)$. Thus we can certainly find $b \in B(x)$ and $b^{\prime} \in B(z)$ such that $b(y H)=m$ and $b\left(x^{-1} y H\right)=n$. If $c \in B(x)$ and $c^{\prime} \in B(z)$ also satisfy $c(y H)=m$ and $c^{\prime}\left(x^{-1} y H\right)=n$, then there are $d \in I(y \cdot H) \cdot B(x)$ and $d^{\prime} \in I\left(x^{-1} y H\right) \cdot B(z)$ such that $c=b+d$ and $c^{\prime}=b^{\prime}+d^{\prime}$. Then

$$
\begin{aligned}
c c^{\prime} & =b b^{\prime}+d b^{\prime}+b d^{\prime}+b^{\prime} d^{\prime} \\
\in b b^{\prime} & +I(y H) B(x) B(z)+B(x) I\left(x^{-1} y H\right) B(z) \\
& +I(y H) B(x) I\left(x^{-1} y H\right) B(z)
\end{aligned}
$$

which, in view of Corollary 8 as well as the observations that $B(x) B(z)=$ $B(x z)$ and $I(y H)^{2}=I(y H)$, is in

$$
b b^{\prime}+I(y H) \cdot B(x z) .
$$

Therefore $c c^{\prime}(y H)=b b^{\prime}(y H)$ in $B(x z) / I(y H) \cdot B(x z)$. Therefore multiplication is well-defined. A similar argument holds for the involution.

To establish continuity of multiplication, we first need to observe that if $b_{i} \rightarrow b$ in $\mathscr{B}$ and if $y_{i} H \rightarrow y H$, then

$$
b_{i}\left(y_{i} H\right) \rightarrow b(y H) \quad \text { in } \mathscr{Q} \text {. }
$$

To see this, let $p\left(b_{i}\right)=x_{i}$ and $p(b)=x$, and let $f \in \Gamma_{c}(G ; \mathscr{B})$ be such that $f(x)=b$. Since $f\left(x_{i}\right)-b_{i} \rightarrow 0_{x}$, we must have

$$
\left\|f\left(x_{i}\right)-b_{i}\right\| \rightarrow 0
$$

But then

$$
\left\|\Phi(f)\left(x_{i}, y_{i} H\right)-b_{i}\left(y_{i} H\right)\right\| \rightarrow 0 .
$$


Since $\Phi(f)\left(x_{i}, y_{i} H\right) \rightarrow \Phi(f)(x, y H)=b(y H)$, it follows from [10, Proposition C.20] that (6) holds.

Now suppose that $\left(c_{i}, c_{i}^{\prime}\right) \rightarrow\left(c, c^{\prime}\right)$ in $\mathscr{Q}^{(2)}$ with

$$
\begin{aligned}
\left(\bar{p}\left(c_{i}\right), \bar{p}\left(c_{i}^{\prime}\right)\right)=\left(\left(x_{i}, y_{i} H\right)\right. & \left.,\left(z_{i}, x_{i}^{-1} y_{i} H\right)\right) \\
& \rightarrow\left(\bar{p}(c), \bar{p}\left(c^{\prime}\right)\right)=\left((x, y H),\left(z, x^{-1} y H\right)\right) .
\end{aligned}
$$

We want to show that $c_{i} c_{i}^{\prime} \rightarrow c c^{\prime}$.

Keep in mind that if $f \in \Gamma_{c}(G ; \mathscr{B})$, then with our conventions, $f(x)(y H)$ and $\Phi(f)(x, y H)$ both denote the image of $f(x)$ in $Q(x, y H)$. In particular, if $f, g \in \Gamma_{c}(G ; \mathscr{B})$, then $(f(x) g(z))(y H)=\Phi(f)(x, y H) \Phi(g)\left(z, x^{-1} y H\right)$. Thus if $f, g \in \Gamma_{c}(G ; \mathscr{B})$ are such that $\Phi(f)(x, y H)=c$ and $\Phi(g)\left(z, x^{-1} y H\right)$ $=c^{\prime}$, then $f\left(x_{i}\right) g\left(z_{i}\right) \rightarrow f(x) g(z)$ in $\mathscr{B}$. Then it follows from (6) that

$$
\Phi(f)\left(x_{i}, y_{i} H\right) \Phi(g)\left(z_{i}, x_{i}^{-1} y_{i} H\right) \rightarrow \Phi(f)(x, y H) \Phi(g)\left(z, x^{-1} y H\right) .
$$

Since

$$
\left\|\Phi(f)\left(x_{i}, y_{i} H\right)-c_{i}\right\| \rightarrow 0 \quad \text { and } \quad\left\|\Phi(g)\left(z_{i}, x_{i}^{-1} y_{i} H\right)-c_{i}^{\prime}\right\| \rightarrow 0,
$$

we can use $[4$, Lemma 1$]$ to show that

$$
\left\|\Phi(f)\left(x_{i}, y_{i} H\right) \Phi(g)\left(z_{i}, x_{i}^{-1} y_{i} H\right)-c_{i} c_{i}^{\prime}\right\| \rightarrow 0 .
$$

Then [10, Proposition C.20] implies that $c_{i} c_{i}^{\prime} \rightarrow c c^{\prime}$. Therefore, multiplication is continuous. The continuity of the involution is proved similarly.

It now follows easily that axioms (a), (b) and (c) of [7, Definition 1.1] are satisfied. Furthermore, if $(e, y H) \in(G \times G / H)^{(0)}$, then $Q(e, y H)$ is the $C^{*}$ algebra $A(y H)$. So axiom (d) is also satisfied. And we have already observed that $Q(x, y H)$ is a $A(y H)-A\left(x^{-1} y H\right)$-imprimitivity bimodule. Thus all the axioms of [7, Definition 1.1] are satisfied and $\bar{p}: \mathscr{Q} \rightarrow G \times G / H$ is a Fell bundle as required.

To verify the last assertion, we observe that $I(e H)=I$ and $Q(h, e H)=$ $B(h) / B(h) \cdot I=C^{I}(h)$. Thus, both $\mathscr{Q}_{\underline{H}}$ and $\mathscr{C}^{I}$ are built from

$$
\coprod_{h \in H} C^{I}(h) .
$$

Therefore it suffice to see that the identity map is a homeomorphism. But in any Banach bundle $p: \mathscr{A} \rightarrow X$, we have $a_{i} \rightarrow a$ in $\mathscr{A}$ if and only if for some section $g \in \Gamma_{c}(X ; \mathscr{A})$ with $g(p(a))=a$, we have $\left\|a_{i}-g\left(p\left(a_{i}\right)\right)\right\| \rightarrow$ 0 (for example, see [7, Lemma A.3]). But if $b \in C^{I}\left(h_{0}\right)$, then there is a $f \in \Gamma_{c c}(G ; \mathscr{B})$ such that $\left[f\left(h_{0}\right)\right]=b$. But $h \mapsto[f(h)]$ is a section of $\mathscr{C}^{I}$ 
and $h \mapsto \Phi(f)(h, e H)=[f(h)]$ is also a section of $\left.2\right|_{\underline{H}}$. It now follows easily that the identity map is bicontinuous. This completes the proof of the proposition.

Lemma 14. We have that $\Gamma_{c c}(G ; \mathscr{B})$ is dense in $\Gamma_{c}(G ; \mathscr{B})$ in the inductive limit topology.

PROoF. This is a straightforward consequence of [7, Lemma A.4] and (4).

Proposition 15. The map $\Phi: \Gamma_{c c}(G ; \mathscr{B}) \rightarrow \Gamma_{c}(G \times G / H ; 2)$ extends to an isomorphism of $C^{*}(G, \mathscr{B})$ onto $C^{*}(G \times G / H, \mathscr{2})$.

Proof. First we'll show that $\Phi$ is a $*$-homomorphism. Then we'll see that $\Phi$ is a bijection of $\Gamma_{c c}(G ; \mathscr{B})$ onto $\Gamma_{c}(G \times G / H ; \mathscr{Q})$. We'll finish by showing that $\Phi$ and $\Phi^{-1}$ are bounded with respect to the universal norms.

Since $b \mapsto b(y H)$ is a bounded linear map of $B(x)$ onto the quotient $B(x) / I(y H) \cdot B(x)$,

$$
\begin{aligned}
f * g(x)(y H) & =\int_{G}\left(f(z) g\left(z^{-1} x\right)\right)(y H) d z \\
& =\int_{G} f(z)(y H) g\left(z^{-1} x\right)\left(z^{-1} y H\right) d z .
\end{aligned}
$$

Therefore, $\varphi(f * g)(x, y H)=\Phi(f) * \Phi(g)(x, y H)$, and $\Phi$ preserves multiplication. Similarly,

$$
\begin{aligned}
\Phi(f)^{*}(x, y H) & =\Phi(f)\left(x^{-1}, x^{-1} y H\right)^{*}=\left(f\left(x^{-1}\right)\left(x^{-1} y H\right)\right)^{*} \\
& =f\left(x^{-1}\right)^{*}(y H),
\end{aligned}
$$

while on the other hand,

$$
\Phi\left(f^{*}\right)(x, y H)=f^{*}(x)(y H)=f\left(x^{-1}\right)^{*}(y H) .
$$

Thus, $\Phi$ is a $*$-homomorphism. Clearly, $\Phi$ is injective.

Using Lemma 11 , we see that $\Phi\left(\Gamma_{c c}(G ; \mathscr{B})\right)$ is a $C_{0}(G \times G / H)$ module. Thus [7, Lemma A.4] implies that $\Phi\left(\Gamma_{c c}(G ; \mathscr{B})\right)$ is inductive limit dense in $\Gamma_{c}(G \times G / H, 2)$. Thus if $F \in \Gamma_{c}(G \times G / H ; 2)$, then there are $f_{i} \in$ $\Gamma_{c c}(G ; \mathscr{B})$ such that $\Phi\left(f_{i}\right) \rightarrow F$ in the inductive limit topology. For each $x \in G, y H \mapsto F(x, y H)$ is (not necessarily continuous) section of $\bar{p}_{x}: \mathscr{Q}_{x} \rightarrow$ $G / H$ which is uniformly approximated by the continuous sections $y H \mapsto$ $\Phi\left(f_{i}\right)(x, y H)=f_{i}(x)(y H)$. Therefore, by Remark 10, there is a $f(x) \in B(x)$ such that $f(x)(y H)=F(x, y H)$. But $f_{i}$ must converge uniformly to $f$, and it follows that $f \in \Gamma_{c c}(G ; \mathscr{B})$. But then $\Phi(f)=F$. 
Now we notice that

$$
\int_{G}\|\Phi(f)(x, y H)\| d x \leq \int_{G}\|f(x)\| d x .
$$

Thus,

$$
\|\Phi(f)\|_{I} \leq\|f\|_{I}
$$

where the $I$-norms are computed in $\Gamma_{c}(G \times G / H ; \mathscr{Q})$ and $\Gamma_{c}(G ; \mathscr{B})$, respectively. Let $L$ be a faithful representation of $C^{*}(G \times G / H, \mathscr{2})$. Then $L \circ \Phi$ is a $I$-norm decreasing $*$-homomorphism of $\Gamma_{c c}(G ; \mathscr{B})$, which must extend to a $I$-norm decreasing representation $L^{\prime}$ of $\Gamma_{c}(G ; \mathscr{B})$ since $\Gamma_{c c}(G ; \mathscr{B})$ is $I$-norm dense in $\Gamma_{c}(G ; \mathscr{B})$ by Lemma 14 . Therefore

$$
\|\Phi(f)\|=\|L(\Phi(f))\|=\left\|L^{\prime}(f)\right\| \leq\|f\|,
$$

and $\Phi$ is norm decreasing for the universal norms.

But if $\Phi\left(f_{i}\right) \rightarrow \Phi(f)$ in the inductive limit topology on $\Gamma_{c}(G \times G / H$; $)$, then $f_{i} \rightarrow f$ in the inductive limit topology on $\Gamma_{c}(G ; \mathscr{B})$. Thus if $R$ is a faithful representation of $C^{*}(G, \mathscr{B})$, then $R \circ \Phi^{-1}$ is a $*$-homomorphism $R^{\prime}$ of $\Gamma_{c}(G \times G / H ; \mathscr{Q})$ which is continuous in the inductive limit topology. By [7, Remark 4.14], $R^{\prime}$ is bounded and

$$
\|f\|=\|R(f)\|=\left\|R^{\prime}(\Phi(f))\right\| \leq\|\Phi(f)\| .
$$

This completes the proof.

\section{REFERENCES}

1. Fell, James M. G., and Doran, Robert S., Representations of *-Algebras, Locally Compact Groups, and Banach *-algebraic Bundles I. Basic Representation Theory of Groups and Algebras, Pure Appl. Math. 125, Academic Press, Boston, 1988.

2. Fell, James M. G., and Doran, Robert S., Representations of $*$-Algebras, Locally Compact Groups, and Banach *-algebraic Bundles II. Banach *-Algebraic Bundles, Induced Representations, and the Generalized Mackey Analysis, Pure Appl. Math. 126, Academic Press, Boston 1988.

3. Green, Philip, The local structure of twisted covariance algebras, Acta Math. 140 (1978), $191-250$

4. Ionescu, Marius, and Williams, Dana P., Remarks on the ideal structure of Fell bundle $C^{*}$ algebras, preprint 2009, (arXiv:math.OA.0912.1124).

5. Kaliszewski, Steven, Muhly, Paul S., Quigg, John, and Williams, Dana P., Coactions and Fell bundles, New York J. Math. 16 (2010), 315-359.

6. Muhly, Paul S., Renault, Jean N., and Williams, Dana P., Equivalence and isomorphism for groupoid $C^{*}$-algebras, J. Operator Theory 17 (1987), 3-22.

7. Muhly, Paul S., and Williams, Dana P., Equivalence and disintegration theorems for Fell bundles and their $C^{*}$-algebras, Dissertationes Math. (Rozprawy Mat.) 465 (2008), 1-57. 
8. Raeburn, Iain, and Williams, Dana P., Morita Equivalence and Continuous-trace C $^{*}$-algebras, Math. Surveys Monogr. 60, Amer. Math. Soc., Providence, RI 1998.

9. Ramsay, Arlan, The Mackey-Glimm dichotomy for foliations and other Polish groupoids, J. Funct. Anal. 94 (1990), 358-374.

10. Williams, Dana P., Crossed Products of $C^{*}$-algebras, Math. Surveys Monogr. 134, Amer. Math. Soc., Providence, RI 2007.

DEPARTMENT OF MATHEMATICS

UNIVERSITY OF CONNECTICUT

STORRS, CT 06269-3009

U.S.A.

E-mail: ionescu@math.uconn.edu

Current address:

DEPARTMENT OF MATHEMATICS

COLGATE UNIVERSITY

HAMILTON, NY 13346

U.S.A.
DEPARTMENT OF MATHEMATICS

DARTMOUTH COLLEGE

HANOVER, NH 03755-3551

U.S.A.

E-mail: dana.williams@Dartmouth.edu 